ISSN 2076-0817

www.mdpi.com/journal/pathogens

Article

\title{
Interaction of Phenol-Soluble Modulins with Phosphatidylcholine Vesicles
}

\author{
Anthony C. Duong, Gordon Y. C. Cheung and Michael Otto * \\ Pathogen Molecular Genetics Section, Laboratory of Human Bacterial Pathogenesis, \\ National Institute of Allergy and Infectious Diseases, The National Institutes of Health, \\ 9000 Rockville Pike, Bethesda, MD 20892, USA; E-Mails: anthony.duong@nih.gov (A.C.D.); \\ cheunggo@niaid.nih.gov (G.Y.C.C.) \\ * Author to whom correspondence should be addressed; E-Mail: motto@ niaid.nih.gov; \\ Tel.: +1-301-443-5209; Fax: +1-301-480-3633.
}

Received: 10 May 2012; in revised form: 5 June 2012 / Accepted: 19 July 2012 /

Published: 20 July 2012

\begin{abstract}
Several members of the staphylococcal phenol-soluble modulin (PSM) peptide family exhibit pronounced capacities to lyse eukaryotic cells, such as neutrophils, monocytes, and erythrocytes. This is commonly assumed to be due to the amphipathic, $\alpha$-helical structure of PSMs, giving PSMs detergent-like characteristics and allowing for a relatively non-specific destruction of biological membranes. However, the capacities of PSMs to lyse synthetic phospholipid vesicles have not been investigated. Here, we analyzed lysis of synthetic phosphatidylcholine (1-palmitoyl-2-oleoyl-sn-glycero-3phosphocholine, POPC) vesicles by all Staphylococcus aureus and S. epidermidis PSMs. In addition, we investigated the lytic capacities of culture filtrates obtained from different S. aureus PSM deletion mutants toward POPC vesicles. Our results show that all staphylococcal PSMs have phospholipid vesicle-lysing activity and the capacity of S. aureus culture filtrate to lyse POPC vesicles is exclusively dependent on PSMs. Notably, we observed largely differing capacities among PSM peptides to lyse POPC vesicles. Interestingly, POPC vesicle-lytic capacities did not correlate with those previously seen for the lysis of eukaryotic cells. For example, the $\beta$-type PSMs were strongly lytic for POPC vesicles, but are known to exhibit only very low lytic capacities toward neutrophils and erythrocytes. Thus our results also suggest that the interaction between PSMs and eukaryotic membranes is more specific than previously assumed, potentially depending on additional structural features of those membranes, such as phospholipid composition or yet unidentified docking molecules.
\end{abstract}


Keywords: phenol-soluble modulin; Staphylococcus aureus; Staphylococcus epidermidis; toxin; vesicles

\section{Introduction}

Many members of the genus Staphylococcus are important human pathogens. Staphylococcus aureus in particular causes a multitude of frequently severe and life-threatening diseases, with acute disease promoted by a series of secreted toxins and other virulence determinants [1]. Coagulase-negative staphylococci, most notably Staphylococcus epidermidis, are a premier cause of hospital-associated infections on indwelling medical devices [2,3].

While virulence of $S$. aureus is clearly multi-factorial, the phenol-soluble modulin (PSM) peptide family has recently been identified as a key contributor to infection with highly virulent $S$. aureus strains, such as community-associated methicillin-resistant $S$. aureus (CA-MRSA) [4,5]. The PSM $\alpha$ peptides in particular strongly impact the capacity of CA-MRSA strains to lyse human neutrophils and other cell types, and promote skin infection and bacteremia. Less virulent hospital-associated strains characteristically produce smaller amounts of those peptide toxins [6].

In addition to their cytolytic potential, PSM peptides promote inflammatory responses by activating the FPR2 receptor [7]. Furthermore, they contribute to biofilm structuring, detachment, and the systemic dissemination of biofilm-associated infection [8,9]. Moreover, some PSMs may exhibit antimicrobial functions, for example toward Streptococcus pyogenes [10,11].

In contrast to the receptor-mediated pro-inflammatory roles of PSMs, the mechanistic basis of other PSM functions, such as cytolysis and biofilm maturation, is less well understood. Because cytolysis by PSMs is of utmost importance for staphylococcal virulence, the mechanism of PSM cytolytic activity is of key interest. Most likely, cytolysis is driven by the surfactant properties of PSMs, which are assumed to be critical for the formation of pores in cytoplasmic membranes and based on the characteristic amphipathic $\alpha$-helical structure of PSMs. In fact, the PSM $\delta$-toxin has been shown to lyse synthetic vesicles by the formation of short-lived pores [12]. However, other PSMs have never been analyzed for their potentials to lyse phospholipid vesicles.

Phosphatidylcholine (POPC) is a major constituent of human cytoplasmic membranes. Membranes of red and white blood cells for example contain about equal amounts of cholesterol and phospholipids, among which POPC is the most abundant at $30 \%$ or higher of total membrane phospholipid $[13,14]$. Notably, POPC is present mostly in the outer leaflet of the human cytoplasmic membrane [15], and thus a predominant component of the membrane part interacting with exogenous toxins such as PSMs. In contrast, bacterial membranes do not contain cholesterol and have a phospholipid composition strongly different from that of eukaryotic membranes. $S$. aureus membranes for example contain mostly phosphatidylglycerol, diphosphatidylglycerol, and lysylphosphatidylglycerol, but no POPC [16].

Here, we analyzed the interaction of all PSM peptides known in S. aureus and S. epidermidis, and of psm isogenic deletion mutants of a CA-MRSA strain, with synthetic POPC vesicles. Our analyses demonstrate pore-forming activity of all PSM peptides. Importantly, PSM peptides without considerable reported cytolytic activities toward human cells, such as PSM $\beta$ peptides, lysed POPC 
vesicles very efficiently. This indicates that the interaction of PSMs with eukaryotic membranes is governed by more complex interactions than previously believed. The elucidation of such interactions will be an important task of future investigations aimed to answer the key question of why eukaryotic cells are much more susceptible to PSM cytolytic activity than bacterial cells.

\section{Results and Discussion}

To analyze whether and to which degree PSM peptides lyse POPC vesicles, we set up a fluorescein release assay. We tested final PSM peptide concentrations in the range of 0.25 to $2 \mu \mathrm{M}$ and chose 0.5 and $1 \mu \mathrm{M}$ for the final assays, as with those concentrations quantitative differences were most evident. The relative capacities of the peptides to lyse the vesicles were measured over time and curves were fit to a one-phase association. Association constants were determined to express differences in lytic capacities.

\subsection{Lytic Activities of PSM Peptides}

All PSM peptides of $S$. aureus and S. epidermidis (Figure 1) showed considerable potencies to lyse POPC vesicles. S. aureus PSM peptides lysed the POPC vesicles when applied at $0.5 \mu \mathrm{M}$ final concentration in the order $\mathrm{PSM} \beta 1>\mathrm{PSM} \beta 2>\mathrm{PSM} \alpha 4>\mathrm{PSM} \alpha 1>\mathrm{PSM} \alpha 3>\mathrm{PSM} \alpha 2>\delta$-toxin (Figure 2). At $1 \mu \mathrm{M}$ final concentration, the order was similar, with only the different PSM $\alpha$ peptides changing order $(\mathrm{PSM} \beta 1>\mathrm{PSM} \beta 2>\mathrm{PSM} \alpha 1>\mathrm{PSM} \alpha 2>\mathrm{PSM} \alpha 3>\mathrm{PSM} \alpha 4>\delta$-toxin) (Figure 2$)$. The capacities of $S$. epidermidis PSM peptides to lyse the vesicles was in the order PSM $\beta 1>\operatorname{PSM} \delta>$ $\mathrm{PSM} \varepsilon>\delta$-toxin $>\mathrm{PSM} \beta 2>\mathrm{PSM} \alpha$ at both 0.5 and $1 \mu \mathrm{M}$ (Figures 2 and 3 ).

Figure 1. Amino acid sequences of PSM peptides of S. aureus and S. epidermidis. Note all PSM peptides carry an N-terminal N-formyl methionine (fM), as they are secreted without signal peptide. Sa, S. aureus; Se, S. epidermidis. PSM-mec is encoded on a methicillin resistance mobile genetic element present in some strains of $S$. aureus and $S$. epidermidis. PSMs of the $\alpha$-type form an amphipathic $\alpha$-helix. In the longer $\beta$-type PSMs, the C-terminal part contains an amphipathic $\alpha$-helix.

Sa $\delta$-toxin
Se $\delta$-toxin
Sa PSM $\alpha 1$
Sa PSM $\alpha 2$
Sa PSM $\alpha 3$
Sa PSM $\alpha 4$
Se PSM $\alpha$
Se PSM $\delta$
Se PSM 8
Sa/Se PSM-mec
Sa PSM $\beta 1$
Sa PSM 32
Se PSM $\beta 1$
Se PSM 32

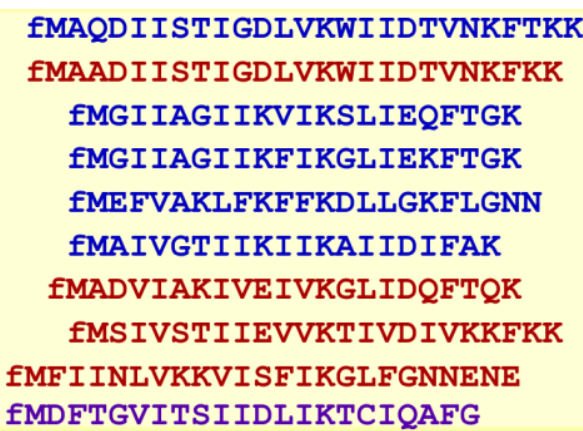

IMEGLFNAIKDTVTAAINNDGAKLGTSIVSIVENGVGLLGKLFGF IMTGLAEAIANTVQAAQQHDSVKLGTSIVDIVANGVGLLGKLFGF IMSKLAEAIANTVKAAQDQDWTKLGTSIVDIVESGVSVLGKIFGF IMEQLFDAIRSVVDAGINQDWSQLASGIAGIVENGISVISKLLGQ 
Notably, the relative capacities of $S$. aureus and $S$. epidermidis PSM peptides to lyse POPC vesicles are thus remarkably different from their capacities to lyse neutrophils and erythrocytes. Neutrophils are lysed by $S$. aureus PSMs in the order PSM $\alpha 3>>$ PSM $\alpha 2$, PSM $\alpha 1, \delta$-toxin $>>$ PSM $\alpha 4$, PSM $\beta 1$, PSM $\beta 2$ and $S$. epidermidis PSMs in the order PSM $\delta>>\mathrm{PSM} \varepsilon>\delta$-toxin $>\mathrm{PSM} \alpha>\operatorname{PSM} \beta 1, \operatorname{PSM} \beta 2[4,17]$. Erythrocytes are lysed by $S$. aureus PSMs in the order PSM $\alpha 3$, PSM $\alpha 2>$ PSM $\alpha 1, \operatorname{PSM} \alpha 4, \operatorname{PSM} \beta 1$, $\operatorname{PSM} \beta 2, \delta$-toxin and $S$. epidermidis PSMs in the order PSM $\delta>>\delta$-toxin, PSM $\alpha, \operatorname{PSM} \varepsilon, \operatorname{PSM} \beta 1>$ PSM $\beta 2[4,17]$. Thus, the strongest differences between lysis of eukaryotic cells and synthetic POPC vesicles were noted for the $\beta$-type peptides of $S$. aureus and S. epidermidis. Namely, while the $\beta$-type peptides were among the most potent to lyse POPC vesicles, they characteristically show almost no potential to lyse neutrophils and only a low potential to lyse erythrocytes [4,17]. Importantly, the latter is in accordance with the apparent lack of influence of PSM $\beta$ peptides on S. aureus skin infection and S. aureus and S. epidermidis bacteremia [4,9].

Figure 2. Lysis of POPC vesicles by $S$. aureus and S. epidermidis PSMs at $0.5 \mu \mathrm{M}$.
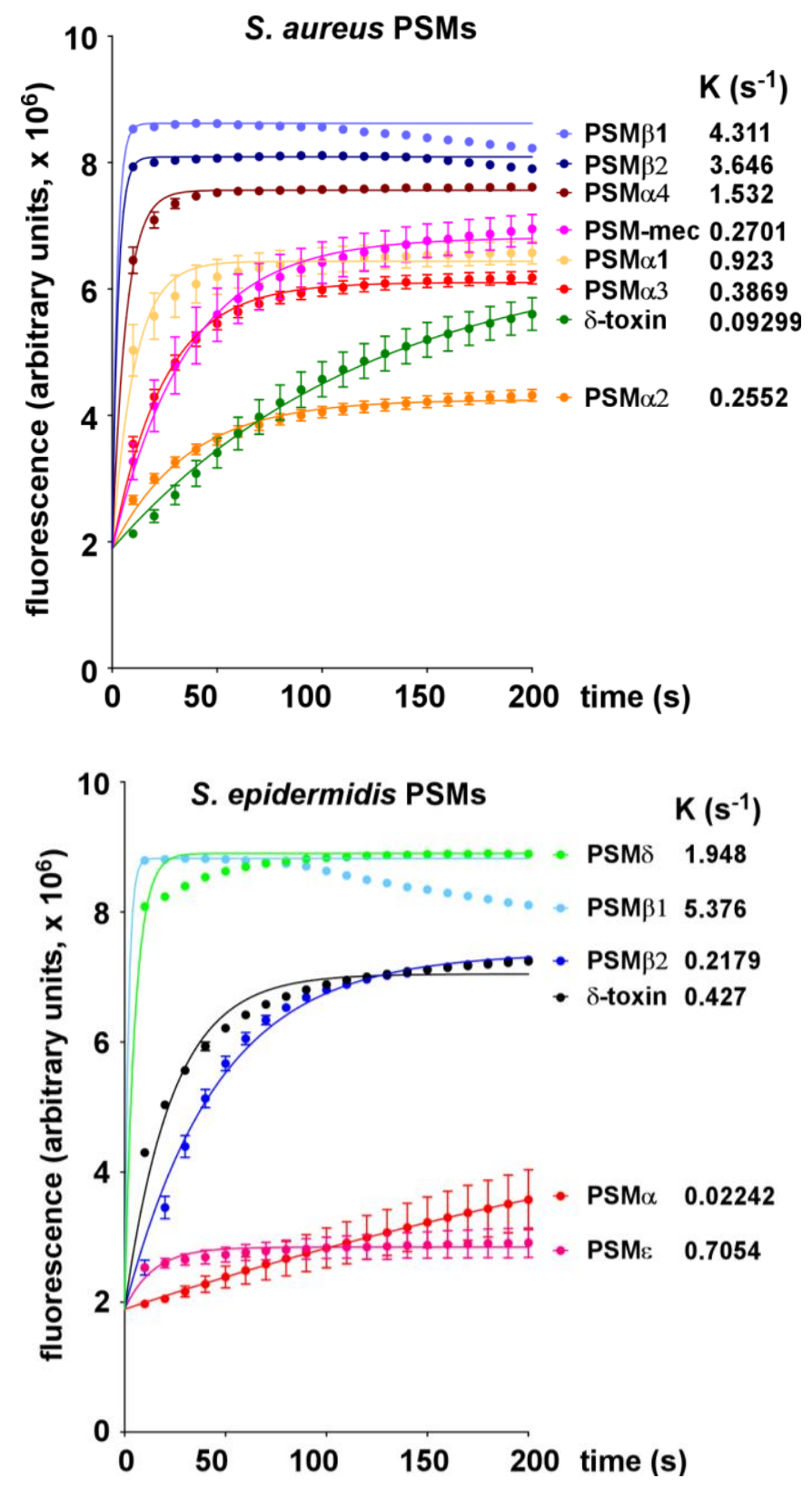
Figure 3. Lysis of POPC vesicles by S. aureus and S. epidermidis PSMs at $1 \mu \mathrm{M}$.
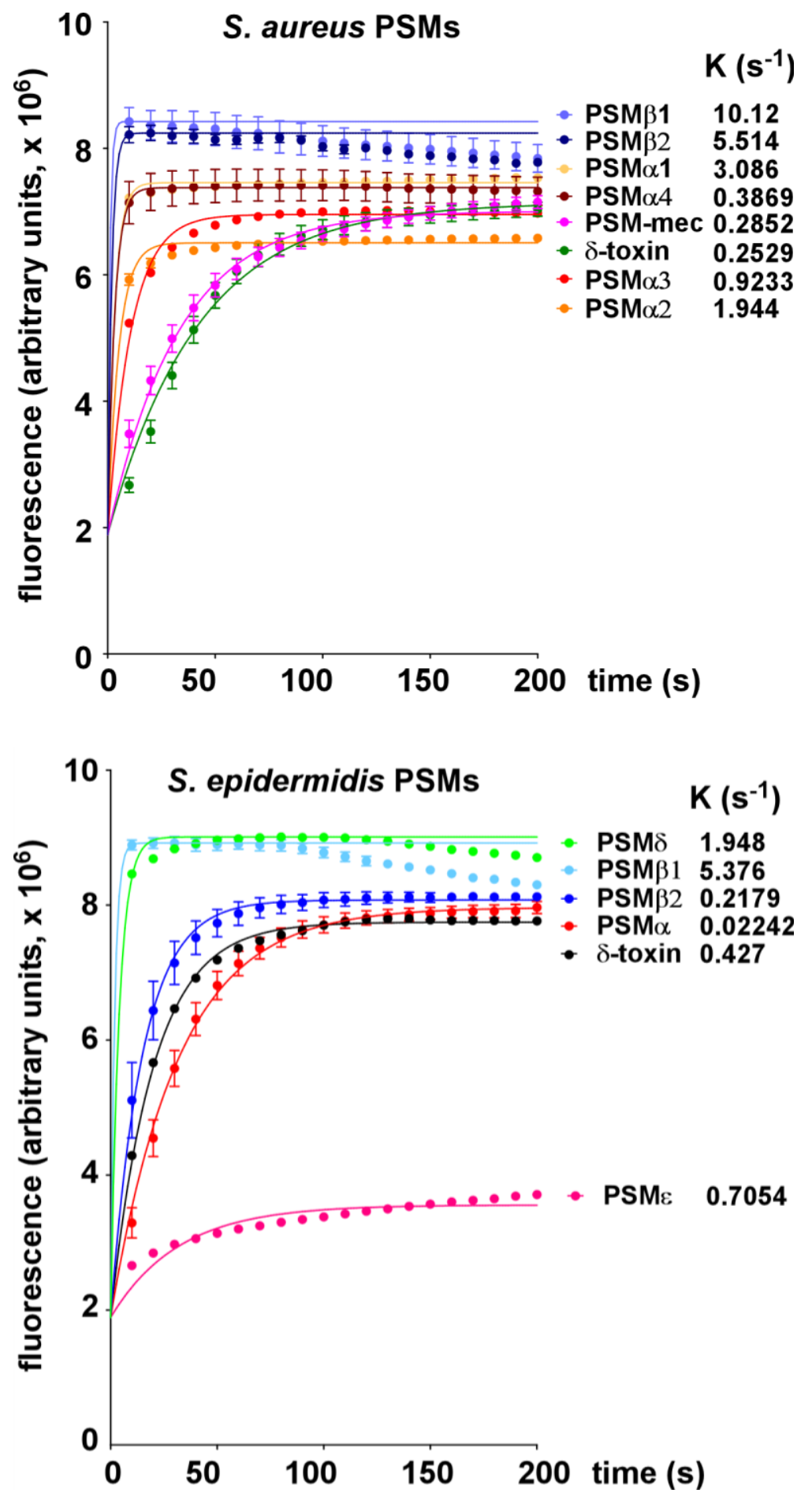

\subsection{Lytic Activities of S. aureus Culture Filtrates}

To analyze POPC-lysing activity of $S$. aureus and its dependence on the secretion of PSM peptides, we analyzed culture filtrates of the CA-MRSA strain LAC (USA300) and isogenic mutants in the $p s m \alpha, p \sin \beta$, or hld ( $\delta$-toxin) loci (Figure 4). We also used a mutant in which sequential deletion of all psm loci resulted in a completely PSM-free S. aureus [11]. Additionally, we analyzed an isogenic mutant in the agr system. Agr strictly regulates production of all PSMs and in agr mutants PSM concentrations are commonly below detection limits $[4,18]$. 
Figure 4. Lysis of POPC vesicles by culture filtrates of $S$. aureus LAC (USA300).

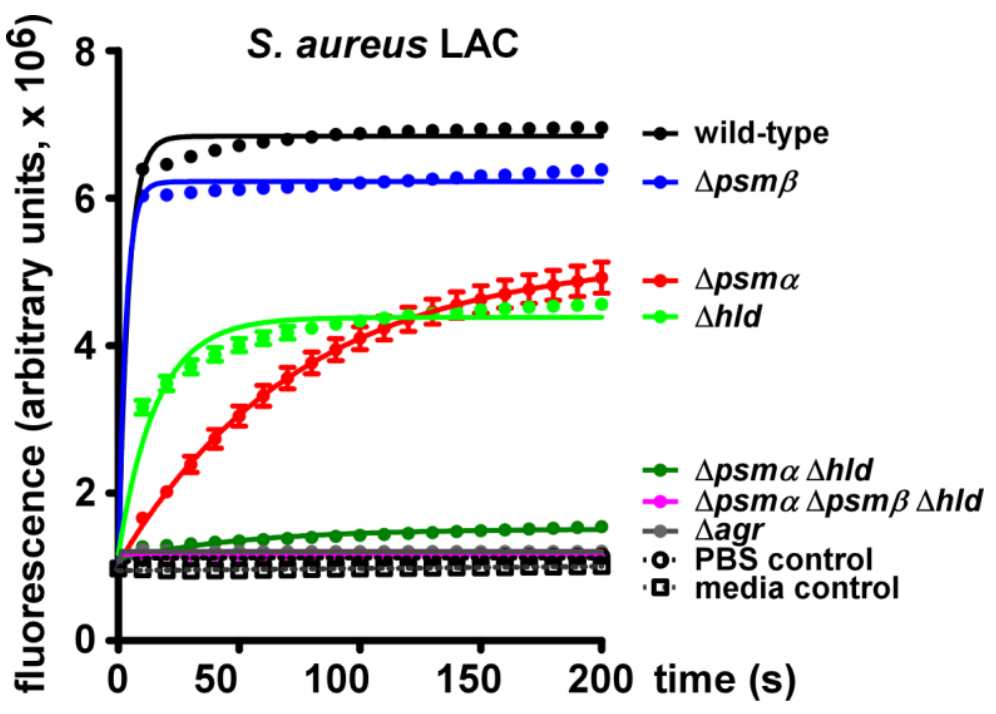

While culture filtrate of the USA300 wild-type strain showed strong capacity to lyse POPC vesicles, lysis was completely abolished when filtrates of the isogenic agr mutant were used. Lytic activity of the agr mutant strain culture filtrate was at the same low level as that observed with PBS or media controls. This finding demonstrates strong capacity of secreted molecules in S. aureus USA300 to lyse POPC vesicles and indicates that all lytic molecules are Agr-regulated.

In addition to PSMs, Agr controls a large series of other secreted proteins, among them several toxins $[19,20]$. Thus, to determine whether the POPC-lytic activity of $S$. aureus USA300 is due to PSMs or other Agr-regulated proteins, we measured the lytic capacity of the PSM-free psma/psm $\beta / h l d$ mutant, in which only PSMs but not other molecules are lacking. This mutant showed a very low level of released fluorescence that was indistinguishable of the levels observed with the agr mutant strain or the controls. Thus, these analyses demonstrated that the POPC-lytic activity of S. aureus USA300 is exclusively due to PSMs.

Next, we assayed psm mutant strains to determine which PSM peptides are primarily responsible for the lytic activity of $S$. aureus culture filtrate. The largest decrease in POPC-lytic activity was seen with culture filtrate of the psm $\alpha$ operon mutant (lacking PSM $\alpha 1, \mathrm{PSM} \alpha 2, \mathrm{PSM} \alpha 3$, and PSM $\alpha 4$ ). Culture filtrate of the $\delta$-toxin mutant also had considerably decreased lytic activity. Contrastingly, lytic activity of the psm $\beta$ operon mutant (lacking PSM $\beta 1$ and PSM $\beta 2$ ) was only slightly decreased compared to that of the wild-type. Accordingly, a mutant in the psma and hld loci (only expressing PSM $\beta 1$ and PSM $\beta 2$ ) showed lytic activity that was only barely increased compared to the total psm deletion and agr culture filtrates, and to negative controls. At first glance, these results appear to contradict those obtained with synthetic PSM peptides (Figures 2,3). However, one has to consider that $\operatorname{PSM} \beta$ peptides are produced at much lower levels compared to PSM $\alpha$ peptides and $\delta$-toxin [21]. Thus, POPC vesicle-lytic activity of $S$. aureus is primarily due to PSM $\alpha$ peptides and, to a somewhat lesser extent, $\delta$-toxin. 


\section{Experimental Section}

1-palmitoyl-2-oleoyl-sn-glycero-3-phosphocholine (POPC) vesicles were prepared as described previously [12]. Briefly, a POPC (Avanti Polar Lipids) lipid film, which was prepared after rotary evaporation and lyophilization, was rehydrated in a solution of $20 \mathrm{mM} 3-(\mathrm{N}$-morpholino)propanesulfonic acid (Sigma), $100 \mathrm{mM}$ potassium chloride (Sigma), $0.01 \mathrm{mM}$ ethylene glycol tetraacetic acid (Sigma), $0.02 \%(\mathrm{w} / \mathrm{v})$ sodium azide, $50 \mathrm{mM}$ carboxyfluorescein (Fluka), and $100 \mathrm{mM}$ potassium chloride (buffer 1). The hydrated lipids were passed through a polycarbonate and nitrocellulose membrane multiple times in a Mini-Extruder (Avanti Polar Lipids, Inc.). Lipid fractions were collected after the extruded material was added to a Sephadex G-25 column equilibrated with buffer 1 without carboxyfluorescein.

Lipid concentrations were measured using the Bartlett assay as described previously [12]. Purified lipid vesicles were diluted to $200 \mu \mathrm{M}$ in equilibration buffer and used within a day of preparation.

Synthetic N-formylated S. aureus and S. epidermidis PSMs (GL Biochem Shanghai Ltd.) were constituted and diluted in dimethyl sulfoxide.

Culture filtrates, from clinical CA-MRSA isolate USA300 (clone LAC) and isogenic psm and $a g r$ deletion mutants, were collected from bacterial cultures in tryptone soy broth (TSB) inoculated 1:100 from pre-cultures grown overnight, and grown for $8 \mathrm{~h}$ at $37{ }^{\circ} \mathrm{C}$, with shaking at $200 \mathrm{rpm}$. PSM production in the culture filtrates was analyzed by high-performance liquid chromatography and confirmed to be as described [11].

Carboxyfluorescein release from POPC lipid vesicles incubated with PSMs or culture filtrates was measured by fluorescence (excitation at $470_{\mathrm{nm}}$, emission at $520_{\mathrm{nm}}$ ) in a 96 well plate fluorimeter (VICTOR3, Perkin Elmer).

Curve fitting was performed using Graph Pad Prism Version 5.04. All assays were performed in triplicate and error bars show \pm SEM.

\section{Conclusions}

Our results demonstrate that all PSM peptides of $S$. aureus and S. epidermidis have the capacity to lyse synthetic phospholipid (POPC) vesicles. Of note, the POPC-lytic activity of $S$. aureus culture filtrate is exclusively dependent on PSMs. Furthermore, we noted considerable differences in the lytic capacities of different PSM peptides, which did not correlate with previously described capacities to lyse eukaryotic cells. These noticeable discrepancies, most pronounced for PSM $\beta$ peptides, indicate that the lytic activities that PSMs have toward human cells is not simply determined by surfactant-type interaction of PSMs with phospholipid bilayers. Elucidation of the molecular basis of these discrepancies, which may include differential composition of bacterial versus eukaryotic membranes or the requirement for specific docking structures, is an important task of future research.

\section{Acknowledgments}

The authors thank Mark Nitz, University of Toronto, for help with vesicle lysis studies. This work was supported by the Intramural Research Program of the National Institute of Allergy and Infectious Diseases (NIAID), U.S. National Institutes of Health (NIH, grant No. ZIA AI000904-11). 


\section{Conflict of Interest}

The authors declare no conflict of interest.

\section{References}

1. Lowy, F.D. Staphylococcus aureus infections. N. Engl. J. Med. 1998, 339, 520-532.

2. Otto, M. Staphylococcus epidermidis - the 'accidental' pathogen. Nat. Rev. Microbiol. 2009, 7, $555-567$.

3. Otto, M. Virulence factors of the coagulase-negative staphylococci. Front. Biosci. 2004, 9, 841-863.

4. Wang, R.; Braughton, K.R.; Kretschmer, D.; Bach, T.H.; Queck, S.Y.; Li, M.; Kennedy, A.D.; Dorward, D.W.; Klebanoff, S.J.; Peschel, A.; et al. Identification of novel cytolytic peptides as key virulence determinants for community-associated MRSA. Nat. Med. 2007, 13, 1510-1514.

5. Kobayashi, S.D.; Malachowa, N.; Whitney, A.R.; Braughton, K.R.; Gardner, D.J.; Long, D.; Bubeck Wardenburg, J.; Schneewind, O.; Otto, M.; DeLeo, F.R. Comparative analysis of USA300 virulence determinants in a rabbit model of skin and soft tissue infection. J. Infect. Dis. 2011, 204, 937-941.

6. Li, M.; Diep, B.A.; Villaruz, A.E.; Braughton, K.R.; Jiang, X.; DeLeo, F.R.; Chambers, H.F.; Lu, Y.; Otto, M. Evolution of virulence in epidemic community-associated methicillin-resistant Staphylococcus aureus. Proc. Natl. Acad. Sci. USA 2009, 106, 5883-5888.

7. Kretschmer, D.; Gleske, A.K.; Rautenberg, M.; Wang, R.; Koberle, M.; Bohn, E.; Schoneberg, T.; Rabiet, M.J.; Boulay, F.; Klebanoff, S.J.; et al. Human formyl peptide receptor 2 senses highly pathogenic Staphylococcus aureus. Cell Host Microbe 2010, 7, 463-473.

8. Periasamy, S.; Joo, H.S.; Duong, A.C.; Bach, T.H.; Tan, V.Y.; Chatterjee, S.S.; Cheung, G.Y.; Otto, M. How Staphylococcus aureus biofilms develop their characteristic structure. Proc. Natl. Acad. Sci. USA 2012, 109, 1281-1286.

9. Wang, R.; Khan, B.A.; Cheung, G.Y.; Bach, T.H.; Jameson-Lee, M.; Kong, K.F.; Queck, S.Y.; Otto, M. Staphylococcus epidermidis surfactant peptides promote biofilm maturation and dissemination of biofilm-associated infection in mice. J. Clin. Invest. 2011, 121, 238-248.

10. Cogen, A.L.; Yamasaki, K.; Sanchez, K.M.; Dorschner, R.A.; Lai, Y.; MacLeod, D.T.; Torpey, J.W.; Otto, M.; Nizet, V.; Kim, J.E.; et al. Selective antimicrobial action is provided by phenol-soluble modulins derived from Staphylococcus epidermidis, a normal resident of the skin. J. Invest. Dermatol. 2010, 130, 192-200.

11. Joo, H.S.; Cheung, G.Y.; Otto, M. Antimicrobial activity of community-associated methicillin-resistant Staphylococcus aureus is caused by phenol-soluble modulin derivatives. J. Biol. Chem. 2011, 286, 8933-8940.

12. Pokorny, A.; Birkbeck, T.H.; Almeida, P.F. Mechanism and kinetics of delta-lysin interaction with phospholipid vesicles. Biochemistry 2002, 41, 11044-11056.

13. Marinetti, G.V.; Cattieu, K. Composition and metabolism of phospholipids of human leukocytes. Chem. Phys. Lipids 1982, 31, 169-177. 
14. Owen, J.S.; McIntyre, N. Erythrocyte lipid composition and sodium transport in human liver disease. Biochim. Biophys. Acta 1978, 510, 168-176.

15. Devaux, P.F.; Morris, R. Transmembrane asymmetry and lateral domains in biological membranes. Traffic 2004, 5, 241-246.

16. Li, M.; Rigby, K.; Lai, Y.; Nair, V.; Peschel, A.; Schittek, B.; Otto, M. Staphylococcus aureus mutant screen reveals interaction of the human antimicrobial peptide dermcidin with membrane phospholipids. Antimicrob. Agents Chemother. 2009, 53, 4200-4210.

17. Cheung, G.Y.; Rigby, K.; Wang, R.; Queck, S.Y.; Braughton, K.R.; Whitney, A.R.; Teintze, M.; DeLeo, F.R.; Otto, M. Staphylococcus epidermidis strategies to avoid killing by human neutrophils. PLoS Pathog. 2010, 6, e1001133.

18. Vuong, C.; Durr, M.; Carmody, A.B.; Peschel, A.; Klebanoff, S.J.; Otto, M. Regulated expression of pathogen-associated molecular pattern molecules in Staphylococcus epidermidis: Quorum-sensing determines pro-inflammatory capacity and production of phenol-soluble modulins. Cell. Microbiol. 2004, 6, 753-759.

19. Recsei, P.; Kreiswirth, B.; O’Reilly, M.; Schlievert, P.; Gruss, A.; Novick, R.P. Regulation of exoprotein gene expression in Staphylococcus aureus by agr. Mol. Gen. Genet. 1986, 202, 58-61.

20. Yao, Y.; Vuong, C.; Kocianova, S.; Villaruz, A.E.; Lai, Y.; Sturdevant, D.E.; Otto, M. Characterization of the Staphylococcus epidermidis accessory-gene regulator response: Quorum-sensing regulation of resistance to human innate host defense. J. Infect. Dis. 2006, 193, 841-848.

21. Cheung, G.Y.; Wang, R.; Khan, B.A.; Sturdevant, D.E.; Otto, M. Role of the accessory gene regulator agr in community-associated methicillin-resistant Staphylococcus aureus pathogenesis. Infect. Immunity 2011, 79, 1927-1935.

(C) 2012 by the authors; licensee MDPI, Basel, Switzerland. This article is an open access article distributed under the terms and conditions of the Creative Commons Attribution license (http://creativecommons.org/licenses/by/3.0/). 